\title{
The role of frailty in geriatric cranial neurosurgery for primary central nervous system neoplasms
}

\author{
Shane Shahrestani, MS, $, 1,2$ Brandon M. Lehrich, BS, ${ }^{3}$ Ali R. Tafreshi, MD, ${ }^{4}$ Nolan J. Brown, BS, ${ }^{5}$ \\ Brian V. Lien, MS, ${ }^{5}$ Seth Ransom, BS, ${ }^{6}$ Ryan C. Ransom, MD, PhD, ${ }^{7}$ Alexander M. Ballatori, BA, ${ }^{1}$ \\ Andy Ton, BS, ${ }^{1}$ Xiao T. Chen, BA, ${ }^{1}$ and Ronald Sahyouni, MD, PhD ${ }^{8}$
}

${ }^{1}$ Keck School of Medicine, University of Southern California, Los Angeles; ${ }^{2}$ Department of Medical Engineering, California Institute of Technology, Pasadena; ${ }^{3}$ Department of Biomedical Engineering, University of California, Irvine, California; ${ }^{4}$ Department of Neurological Surgery, Geisinger Health System, Danville, Pennsylvania; ${ }^{5}$ School of Medicine, University of California, Irvine, California; ${ }^{6}$ College of Medicine, University of Arkansas for Medical Sciences, Little Rock, Arkansas; ${ }^{7}$ Department of Neurological Surgery, Mayo Clinic, Rochester, Minnesota; and ${ }^{8}$ Department of Neurological Surgery, University of California, San Diego, La Jolla, California

OBJECTIVE Frailty is a clinical state of increased vulnerability due to age-associated decline and has been well established as a perioperative risk factor. Geriatric patients have a higher risk of frailty, higher incidence of brain cancer, and increased postoperative complication rates compared to nongeriatric patients. Yet, literature describing the effects of frailty on short- and long-term complications in geriatric patients is limited. In this study, the authors evaluate the effects of frailty in geriatric patients receiving cranial neurosurgery for a primary CNS neoplasm.

METHODS The authors conducted a retrospective cohort study of geriatric patients receiving cranial neurosurgery for a primary CNS neoplasm between 2010 and 2017 by using the Nationwide Readmission Database. Demographics and frailty were queried at primary admission, and readmissions were analyzed at 30-, 90-, and 180-day intervals. Complications of interest included infection, anemia, infarction, kidney injury, CSF leak, urinary tract infection, and mortality. Nearest-neighbor propensity score matching for demographics was implemented to identify nonfrail control patients with similar diagnoses and procedures. The analysis used Welch two-sample t-tests for continuous variables and chi-square test with odds ratios.

RESULTS A total of 6713 frail patients and 6629 nonfrail patients were identified at primary admission. At primary admission, frail geriatric patients undergoing cranial neurosurgery had increased odds of developing acute posthemorrhagic anemia (OR 1.56, 95\% Cl 1.23-1.98; $p=0.00020)$; acute infection (OR 3.16, 95\% Cl 1.70-6.36; $p=0.00022)$; acute kidney injury (OR 1.32, 95\% Cl 1.07-1.62; $p=0.0088)$; urinary tract infection prior to discharge (OR 1.97, 95\% $\mathrm{Cl} 1.71-2.29 ; p<0.0001)$; acute postoperative cerebral infarction (OR 1.57, 95\% Cl 1.17-2.11; $p=0.0026$ ); and mortality (OR 1.64, 95\% Cl 1.22-2.24; $p=0.0012)$ compared to nonfrail geriatric patients receiving the same procedure. In addition, frail patients had a significantly increased inpatient length of stay $(p<0.0001)$ and all-payer hospital cost $(p<$ 0.0001 ) compared to nonfrail patients at the time of primary admission. However, no significant difference was found between frail and nonfrail patients with regard to rates of infection, thromboembolism, CSF leak, dural tear, cerebral infarction, acute kidney injury, and mortality at all readmission time points.

CONCLUSIONS Frailty may significantly increase the risks of short-term acute complications in geriatric patients receiving cranial neurosurgery for a primary CNS neoplasm. Long-term analysis revealed no significant difference in complications between frail and nonfrail patients. Further research is warranted to understand the effects and timeline of frailty in geriatric patients.

https://thejns.org/doi/abs/10.3171/2020.7.FOCUS20426

KEYWORDS geriatric; cranial; tumor; frailty; surgical outcome; risk factor; neurosurgery

ABBREVIATIONS CCI = Charlson Comorbidity Index; JHACG = Johns Hopkins Adjusted Clinical Groups; LOS = length of stay; NRD = Nationwide Readmission Database; $\mathrm{UTI}=$ urinary tract infection

SUBMITTED May 22, 2020. ACCEPTED July 17, 2020

INCLUDE WHEN CITING DOI: 10.3171/2020.7.FOCUS20426. 
$\mathrm{F}$ RAILTY is defined as a clinically recognizable state of increased vulnerability resulting from age-associated physiological decline, and frail patients continue to be at a higher risk of poor health outcomes, including falls, disability, perioperative complications, readmissions, and mortality. ${ }^{1-6}$ The direct relationship between advanced age and frailty makes frailty a particular concern in geriatric and elderly patient populations $(\geq 65$ years old), especially those receiving complex surgical interventions including cranial neurosurgery. ${ }^{7-12}$ However, the literature evaluating the influence of frailty on longterm complications in geriatric patients receiving cranial neurosurgery is limited, and many studies that strive to evaluate frailty in cranial neurosurgery fail to stratify patient populations by surgical indication and demographics prior to analysis. ${ }^{13,14}$

To address this gap in the literature, we used the Nationwide Readmission Database (NRD) to identify a cohort of geriatric patients undergoing cranial neurosurgery for a primary CNS neoplasm. Databases like the NRD allow for nationally representative analysis over a range of years with sufficient information to query postoperative complications within a single calendar year. Through this study, we aimed to identify potential complications that may disproportionately affect frail patients. Such an understanding would facilitate the identification of high-risk patient conditions and allow providers to tailor clinical management of frail geriatric patients to minimize postoperative complications.

\section{Methods \\ Data Source}

In this study we used the Healthcare Cost and Utilization Project (HCUP) NRD from the years 2010 to 2017. The NRD is a large yearly database that publishes national information regarding inpatient demographics, diagnoses, procedures, and readmissions. Patient hospital admissions are de-identified and are each represented as unique patient linkages to allow for accurate patient tracking throughout the calendar year. The NRD is publicly available for purchase and has been designed to allow for nationally representative readmission analysis when used in conjunction with the provided NRD discharge weights. Between all years of the NRD included in this study, we identified more than 100 million patient discharges, and all data regarding patient diagnoses and procedures were queried using International Classification of Diseases, Ninth Revision (ICD-9) and International Classification of Diseases, Tenth Revision (ICD-10) codes in all patient admissions and readmissions. Using relevant cost-tocharge ratios provided with the NRD, which are imputed from national hospital-specific or hospital group averaged all-payer inpatient cost data, we converted hospital charges to all-payer inpatient costs. Institutional Review Board approval and informed consent were not required because we used a de-identified, publicly available database.

\section{Patient Selection and Analysis}

Throughout all 8 years, a total of 447,469 patients with benign or malignant primary CNS neoplasms were identi- fied using appropriate ICD coding (Supplementary Table 1). Nongeriatric patients ( $<65$ years old) and all patients who did not receive open cranial neurosurgery for resection of a primary CNS neoplasm at the primary admission were excluded from analysis, leaving 33,836 patients (Fig. 1). Of these patients, 6713 (19.8\%) were found to have ICD9 or ICD-10 codes for frailty according to the Johns Hopkins Adjusted Clinical Groups (JHACG) frailty-defining diagnosis indicator. ${ }^{15-17}$ The JHACG frailty-defining diagnosis indicator uses a set of 10 clinical clusters (malnutrition, dementia, vision impairment, decubitus ulcer, urine control, weight loss, fecal control, social support, difficulty walking, and history of a fall) to predict patient frailty and has been validated through many studies with various patient populations. Charlson Comorbidity Index (CCI) scores were collected for each patient and used to develop 10 -year survival estimates for all patients. ${ }^{18-20}$ Both CCI and 10-year survival were developed by Charlson et al. in their 1987 publication and have since been validated through several subsequent studies. ${ }^{18-22}$

Secondary metastatic neoplasms to the brain were not queried for analysis. Nearest-neighbor propensity score matching for age, sex, and CCI was performed using the R "MatchIt" algorithm, resulting in a cohort of 6629 nonfrail control patients. ${ }^{23}$ In this technique, parametric models are chosen based on the minimum "distance" parameter, which is determined through logistic regression models that minimize the propensity score with no replacement. MatchIt improves parametric statistical models and reduces model dependence by preprocessing data with semiparametric and nonparametric matching methods. Model balance, defined as the similarity of empirical covariate distributions between the two groups undergoing propensity matching, is analyzed and the model with the best balance is selected to ensure best model fit (Fig. 2). Relevant postoperative complications, including acute posthemorrhagic anemia, short-term and long-term infection, dural tear, CSF leaks, thromboembolism, cerebral ischemia, acute kidney injury, and mortality, were compared between frail and propensity score-matched nonfrail cohorts. Readmission was queried at 30-, 90-, and 180-day intervals with appropriate analysis of complications, and all individuals lacking full follow-up at each time point were excluded from analysis.

\section{Statistical Analysis}

All statistics included in this analysis were conducted in RStudio (version 1.2.5042). All statistical tests were two-sided and used an $\alpha=0.05$ level of significance. Following propensity score matching, univariate Welch twosample t-tests were used to compare continuous data elements between frail and nonfrail patient cohorts. These values are expressed as the mean $\pm \mathrm{SD}$. Chi-square testing with post hoc odds ratio calculations were also performed using the R "Epitools" package on binarized data elements between the two cohorts.

\section{Results \\ Demographics}

The average age of the frail cohort was $73.7 \pm 6.2$ years, 


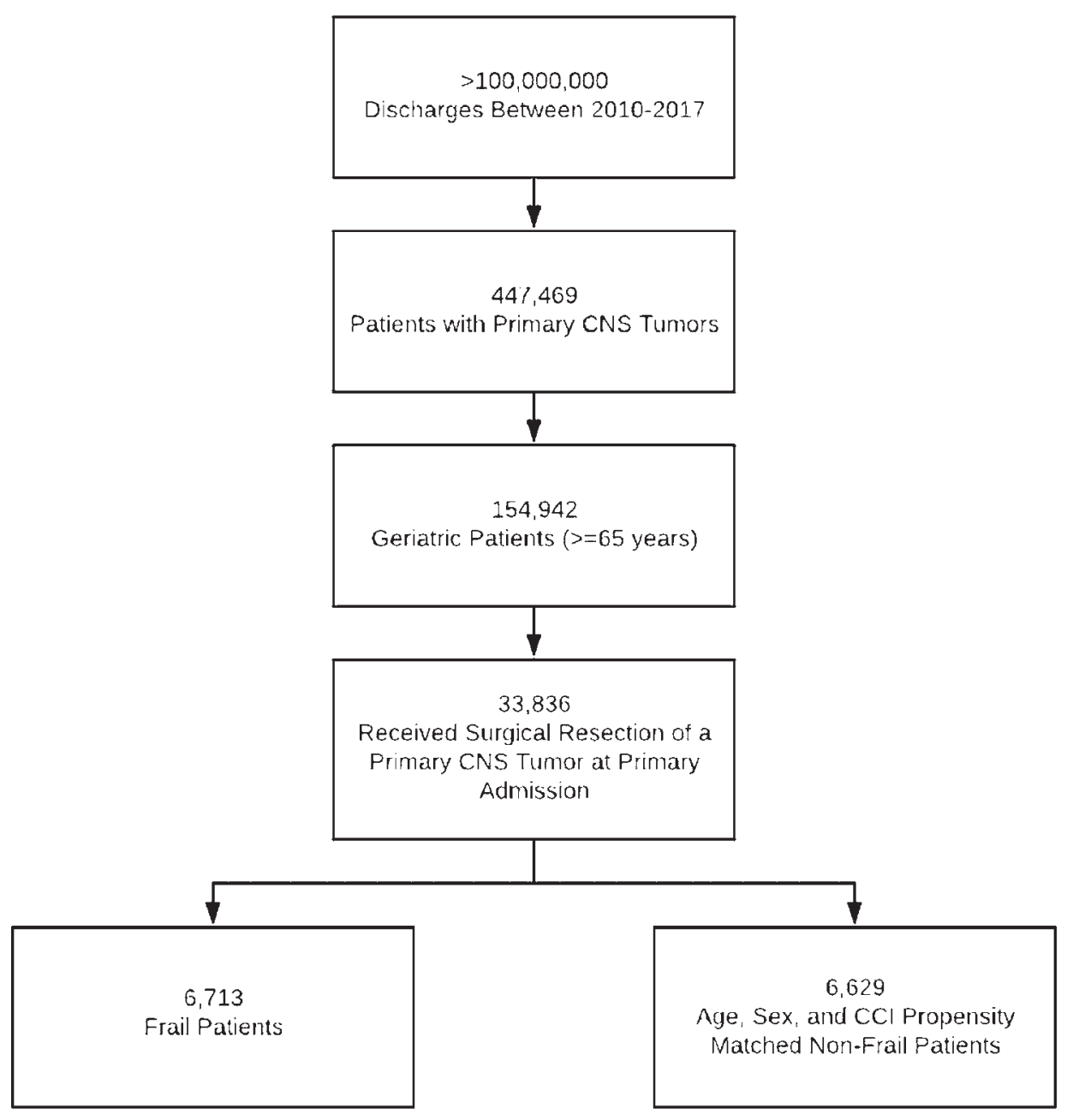

FIG. 1. Patient selection flowchart.

$45.7 \%$ were female, and the average CCI was $6.1 \pm 2.6$, with an estimated 10-year survival rate of $24.0 \%$. The average age of the propensity-matched nonfrail cohort was $73.6 \pm 6.1$ years, $45.2 \%$ were female, and the average CCI was $6.1 \pm 2.7$, with an estimated 10 -year survival rate of $25.0 \%$. No statistically significant difference was found between age, sex, CCI, and 10-year survival as a result of propensity score matching. Furthermore, statistical analysis revealed no difference in insurance type, hospital teaching status, and hospital bed size between the frail and nonfrail cohorts. However, comparison of the median household income quartile by zip code revealed that frail patients are more likely to have a higher household income compared to nonfrail patients $(\mathrm{p}=0.034)$ (Table 1$)$.

\section{Primary Admission}

Within the frail cohort, $286(4.3 \%)$ were diagnosed with a benign CNS neoplasm, 6418 (95.6\%) were diagnosed with a malignant CNS neoplasm, and $9(0.1 \%)$ were found to have both benign and malignant CNS neoplasms present. Within the propensity score-matched nonfrail cohort, 211 (3.2\%) were diagnosed with a benign CNS neoplasm,
$6418(96.7 \%)$ were diagnosed with a malignant CNS neoplasm, and $11(0.1 \%)$ were found to have both benign and malignant CNS neoplasms present. The average inpatient length of stay (LOS) of frail patients was $16.1 \pm 13.9$ days and was found to be significantly higher than the LOS of nonfrail patients, which was $9.0 \pm 8.1$ days $(\mathrm{p}<0.0001)$. Analysis of inpatient all-payer hospital costs between frail and nonfrail patients revealed that frailty was associated with a significantly higher cost (frail: $\$ 39,114.69$ $\pm \$ 38,249.02$; nonfrail: $\$ 27,924.03 \pm \$ 23,886.26[\mathrm{p}<$ $0.0001])$. During the primary admission, frail geriatric patients undergoing cranial neurosurgery had increased odds of developing acute posthemorrhagic anemia (OR $1.56,95 \%$ CI 1.23-1.98; $\mathrm{p}=0.00020)$; acute postoperative infection (OR 3.16, 95\% CI 1.70-6.36; $\mathrm{p}=0.00022$ ); acute kidney injury (OR 1.32, 95\% CI 1.07-1.62; p = 0.0088); urinary tract infection (UTI) prior to initial discharge (OR 1.97, 95\% CI 1.71-2.29; p < 0.0001); acute postoperative cerebral infarction (OR 1.57, 95\% CI 1.17-2.11; $\mathrm{p}=$ 0.0026 ); and mortality (OR 1.64, 95\% CI 1.22-2.24; $\mathrm{p}=$ $0.0012)$ compared to nonfrail geriatric patients receiving the same procedure. As a result, frail patients had a higher 


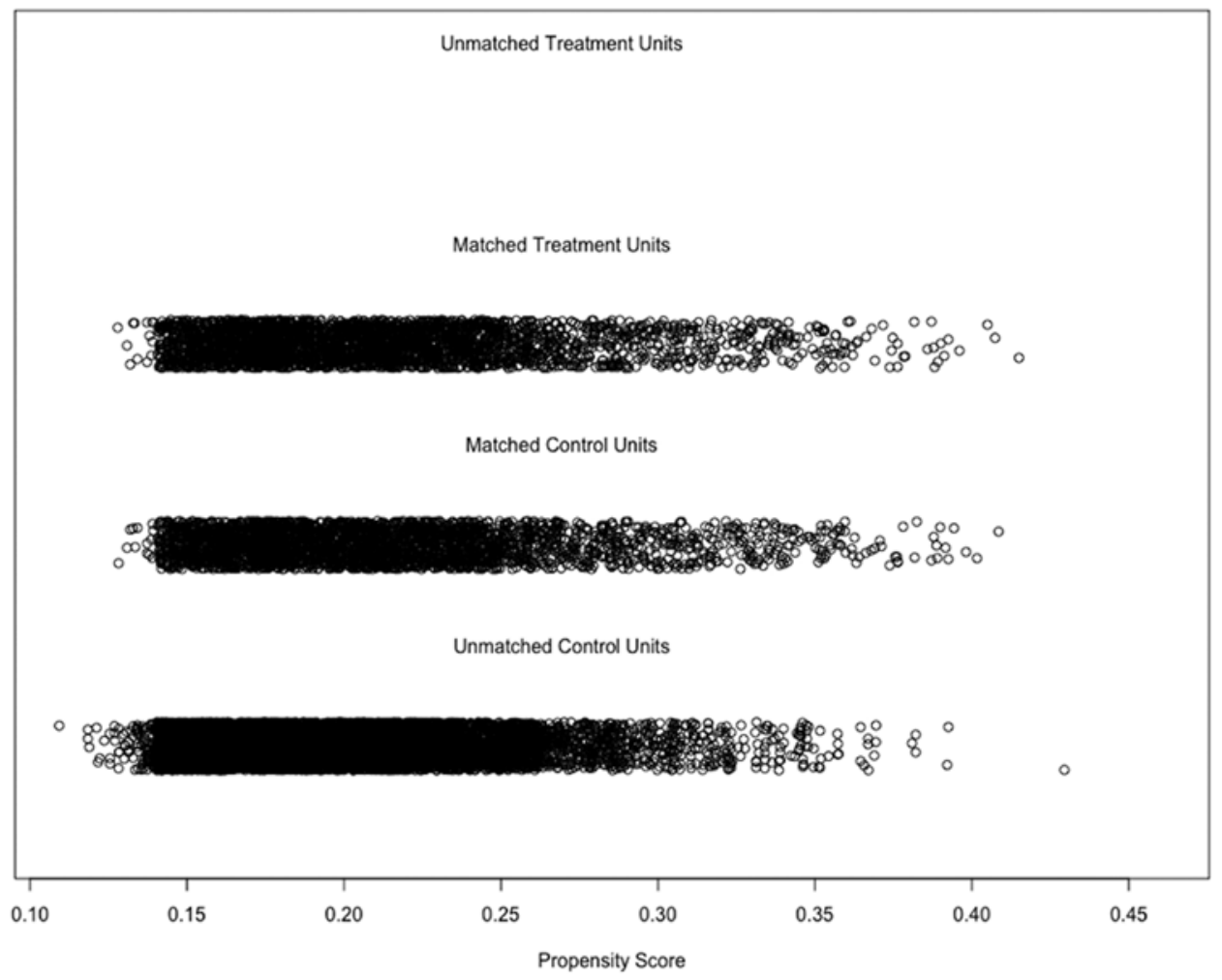

FIG. 2. Distribution of propensity scores following one-to-one nearest-neighbor matching. As seen in the distribution of matched treatment and control units, excellent propensity score matching was achieved with no unmatched treatment units. The unmatched control unit cohort had lower propensity scores, on average, compared to our treatment (frail) cohort. As such, propensity score matching was necessary to ensure that our statistical analysis included two cohorts with similar demographics and comorbidities.

rate of nonroutine hospital discharges, which encompasses transfers to skilled nursing home facilities, short-term hospitals, and home health care, compared to nonfrail patients (OR 1.88, 95\% CI 1.70-2.09; p < 0.0001) (Table 2).

\section{Readmission and Complications}

Readmissions were queried at 30-, 90-, and 180-day intervals and complications were analyzed at each time point. The 30-day readmission rate of frail patients was found to be significantly lower than that of nonfrail patients (OR 0.79, 95\% CI 0.68-0.93; p = 0.0045), but no significant difference in readmission rate was found at 90 or 180 days between the two cohorts. Furthermore, no significant difference was found between frail and nonfrail patients with regard to rates of infection, thromboembolism, CSF leak, dural tear, cerebral infarction, acute kidney injury, and mortality at 30-, 90-, and 180-day readmission (Table 3).

\section{Discussion}

In this 8-year retrospective cohort analysis, we evaluated the influence of patient frailty on postoperative complications in geriatric patients receiving cranial neurosurgery for a primary CNS neoplasm. By comparing a cohort of frail geriatric patients to a propensity-matched cohort of nonfrail geriatric patients, we found that although a sig- nificantly higher proportion of frail patients had a higher median household income, they continued to have worse outcomes immediately following surgery. Namely, frail patients were found to have significantly increased LOS and all-payer hospital costs and higher rates of acute posthemorrhagic anemia, infection, acute kidney injury, UTI, cerebral infarction, mortality, and nonroutine hospital discharge compared to a demographically matched nonfrail cohort receiving the same operation. However, the nonfrail cohort showed a higher 30-day readmission rate and no significant differences in complication rates were found at 30-, 90-, and 180-day readmission intervals.

Although it is well understood that geriatric patients are at a higher risk for frailty and perioperative complications, few studies in the neurosurgical literature have analyzed these factors with respect to patient age and surgical indication. Specifically, a majority of neurosurgical studies that assess frailty fail to comment on the effects of frailty on geriatric patients in particular, and the conclusions drawn from these studies are too broad to guide the management of geriatric patients. ${ }^{13,14,24-26}$ Yet, improvements in neurosurgical management of geriatric patients with primary CNS neoplasms are necessary because geriatric patients have a higher incidence of brain cancer, display a markedly different constellation of presenting symptoms, and have increased complication rates compared to nongeriatric patients..$^{27,28}$ Through subgrouping, age stratifica- 
TABLE 1. Demographics of frail and nonfrail patients

\begin{tabular}{|c|c|c|c|}
\hline Variable & $\begin{array}{c}\text { Frail } \\
\text { Patients, } \\
n=6,713\end{array}$ & $\begin{array}{c}\text { Propensity-Matched } \\
\text { Nonfrail Patients, } \\
n=6,629\end{array}$ & $p$ Value \\
\hline $\begin{array}{l}\text { Age in yrs, mean } \\
\pm S D\end{array}$ & $73.7 \pm 6.2$ & $73.6 \pm 6.1$ & 0.94 \\
\hline \multicolumn{4}{|l|}{ Sex } \\
\hline Female & $3,067(45.7 \%)$ & $2,996(45.2 \%)$ & \multirow{2}{*}{0.88} \\
\hline Male & $3,646(54.3 \%)$ & $3,633(54.8 \%)$ & \\
\hline $\mathrm{CCl}$, mean $\pm \mathrm{SD}$ & $6.1 \pm 2.6$ & $6.1 \pm 2.7$ & 0.86 \\
\hline \multicolumn{4}{|l|}{ Insurance } \\
\hline Medicare & $5,881(87.6 \%)$ & $5,782(87.2 \%)$ & \multirow{4}{*}{0.35} \\
\hline Medicaid & $75(1.1 \%)$ & $58(0.9 \%)$ & \\
\hline Private & $600(8.9 \%)$ & $642(9.7 \%)$ & \\
\hline Self-pay & $19(0.3 \%)$ & $31(0.5 \%)$ & \\
\hline \multicolumn{4}{|l|}{$\begin{array}{l}\text { Median income by } \\
\text { zip code }\end{array}$} \\
\hline Quartile 1 & $1,440(21.5 \%)$ & $1,271(19.2 \%)$ & \multirow{4}{*}{0.034} \\
\hline Quartile 2 & $1,628(24.3 \%)$ & $1,662(25.1 \%)$ & \\
\hline Quartile 3 & $1,785(26.6 \%)$ & $1,674(25.3 \%)$ & \\
\hline Quartile 4 & $1,749(26.1 \%)$ & $1,913(28.9 \%)$ & \\
\hline \multicolumn{4}{|l|}{ Hospital type } \\
\hline $\begin{array}{l}\text { Metropolitan } \\
\text { nonteaching }\end{array}$ & $1,140(17.0 \%)$ & $1,065(16.1 \%)$ & \multirow{3}{*}{0.56} \\
\hline $\begin{array}{l}\text { Metropolitan } \\
\text { teaching }\end{array}$ & $5,432(80.9 \%)$ & $5,462(82.4 \%)$ & \\
\hline Nonmetropolitan & $143(2.1 \%)$ & $102(1.5 \%)$ & \\
\hline \multicolumn{4}{|l|}{ Discharge location } \\
\hline Routine/home & $1,814(27.0 \%)$ & $2,792(42.1 \%)$ & \multirow{2}{*}{$<0.0001$} \\
\hline Nonroutine & $4,899(73.0 \%)$ & $3,837(57.9 \%)$ & \\
\hline
\end{tabular}

Values are expressed as either the mean \pm SD or the number of patients (\%). Boldface type indicates statistical significance at $p<0.05$.

tion, and propensity score matching we aimed to isolate the effects of frailty in geriatric neurosurgical patients with CNS tumors.

In addition, prior studies have demonstrated similar findings with regard to higher rates of short-term complications in frail patients. Cloney et al. have shown that frail geriatric patients with glioblastoma had increased LOS and complication rates and decreased survival compared to nonfrail patients. ${ }^{9}$ However, this study was limited by a small sample size of 319 patients and lack of follow-up data. Similarly, Asemota and Gallia have demonstrated increased short-term complication rates in frail patients receiving transsphenoidal pituitary surgery. ${ }^{14}$ Although this study boasts a large patient sample, the database used for analysis was several decades old and limited follow-up to 30 days, preventing long-term analysis. Thus, the contemporary cohort we present in this study provides novel findings with regard to the short- and long-term effects of frailty in geriatric patients with primary CNS neoplasms.

Moreover, our study found significant differences in complication rates between frail and nonfrail patients immediately following cranial surgery but not at long-term readmission. This finding is supported by previous studies that have demonstrated increased short-term morbidity and mortality in frail patients receiving several different surgical treatments, including cardiothoracic, abdominal, orthopedic, and neurological surgery., ${ }^{9,29-33}$ Although long-term assessments of perioperative complications in frail patients are currently limited, one study by Hewitt et al. found no significant difference in mortality between frail and nonfrail patients receiving general surgery at 90day follow-up. ${ }^{34}$ Whereas the short-term effects of frailty in neurosurgery have been studied, there continues to be a gap in neurosurgical literature with regard to long-term complications in frail versus nonfrail patients. The high rates of acute morbidity and mortality seen in our cohort analysis of frail geriatric patients with primary CNS neoplasms are consistent with previous surgical literature; yet, our study suggests that there may be no significant difference in complications between frail and nonfrail patients at long-term (90 and 180 days) follow-up. In addition, although we report a statistically significant increase in the readmission of nonfrail patients at 30 days, the degree of increase compared to the frail cohort is small (1.7\%), and that is probably due to the sample size included for analysis. As a result, metrics such as LOS, which demonstrated an impressive increase in frail patients compared

TABLE 2. Immediate (acute) postoperative complications

\begin{tabular}{lccccc}
\hline \multicolumn{1}{c}{ Variable } & $\begin{array}{c}\text { Frail Patients, } \\
\mathrm{n}=6,713\end{array}$ & $\begin{array}{c}\text { Propensity-Matched Nonfrail } \\
\text { Patients, } \mathrm{n}=6,629\end{array}$ & OR & $95 \% \mathrm{Cl}$ & $\mathrm{pValue}$ \\
\hline All-payer cost, mean $\pm \mathrm{SD}$ & $\$ 39,114.69 \pm \$ 38,249.02$ & $\$ 27,924.03 \pm \$ 23,886.26$ & $\mathrm{NA}$ & $\mathrm{NA}$ & $<0.0001$ \\
\hline LOS in days, mean $\pm \mathrm{SD}$ & $16.1 \pm 13.9$ & $9.0 \pm 8.1$ & $\mathrm{NA}$ & $\mathrm{NA}$ & $<0.0001$ \\
\hline Acute posthemorrhagic anemia & $443(6.6 \%)$ & $246(3.7 \%)$ & 1.56 & $1.23-1.98$ & $\mathbf{0 . 0 0 0 2 0}$ \\
\hline Infection & $73(1.1 \%)$ & $23(0.3 \%)$ & 3.16 & $1.70-6.36$ & $\mathbf{0 . 0 0 0 2 2}$ \\
\hline Acute kidney injury & $454(6.8 \%)$ & $333(5.0 \%)$ & 1.32 & $1.07-1.62$ & $\mathbf{0 . 0 0 8 8}$ \\
\hline UTI & $1,180(17.6 \%)$ & $654(9.9 \%)$ & 1.97 & $1.71-2.29$ & $<0.0001$ \\
\hline Cerebral infarction & $236(3.5 \%)$ & $160(2.4 \%)$ & 1.57 & $1.17-2.11$ & $\mathbf{0 . 0 0 2 6}$ \\
\hline Mortality & $203(3.0 \%)$ & $138(2.1 \%)$ & 1.64 & $1.22-2.24$ & $\mathbf{0 . 0 0 1 2}$ \\
\hline Nonroutine discharge & $4,899(73.0 \%)$ & $3,837(57.9 \%)$ & 1.88 & $1.70-2.09$ & $<0.0001$ \\
\hline
\end{tabular}

$\mathrm{NA}=$ not applicable.

Values are expressed as either the mean \pm SD or the number of patients (\%). Boldface type indicates statistical significance at $p<0.05$. 
TABLE 3. Postoperative complications at readmission

\begin{tabular}{|c|c|c|c|c|c|}
\hline Timing of Readmission & Frail Patients, $n=6,134$ & Propensity-Matched Nonfrail Patients, $n=6,092$ & OR & $95 \% \mathrm{Cl}$ & p Value \\
\hline \multicolumn{6}{|l|}{ 30-day readmission } \\
\hline Readmission rate & $646(10.5 \%)$ & $742(12.2 \%)$ & 0.79 & $0.68-0.93$ & 0.0045 \\
\hline Infection & $57(0.9 \%)$ & $59(1.0 \%)$ & 1.14 & $0.67-1.93$ & 0.64 \\
\hline Thromboembolism & $69(1.1 \%)$ & $65(1.1 \%)$ & 1.27 & $0.77-2.10$ & 0.35 \\
\hline Acute kidney injury & $98(1.6 \%)$ & $75(1.2 \%)$ & 1.18 & $0.75-1.86$ & 0.47 \\
\hline Dural tear & $0(0.0 \%)$ & $0(0.0 \%)$ & NA & NA & NA \\
\hline CSF leak & $3(0.05 \%)$ & $7(0.1 \%)$ & 0.64 & $0.08-3.50$ & 0.57 \\
\hline Cerebral infarction & $22(0.4 \%)$ & $25(0.4 \%)$ & 1.05 & $0.45-2.41$ & 0.91 \\
\hline \multirow[t]{2}{*}{ Mortality } & $40(0.7 \%)$ & $46(0.8 \%)$ & 1.20 & $0.64-2.24$ & 0.56 \\
\hline & Frail Patients, $n=5,028$ & Propensity-Matched Nonfrail Patients, $n=5,036$ & OR & $95 \% \mathrm{Cl}$ & $\mathrm{p}$ Value \\
\hline \multicolumn{6}{|l|}{ 90-day readmission } \\
\hline Readmission rate & $1,525(30.3 \%)$ & $1,581(31.4 \%)$ & 0.99 & $0.88-1.12$ & 0.92 \\
\hline Infection & $93(1.8 \%)$ & $104(2.1 \%)$ & 0.92 & $0.61-1.36$ & 0.66 \\
\hline Thromboembolism & $180(3.6 \%)$ & $219(4.3 \%)$ & 0.86 & $0.64-1.15$ & 0.31 \\
\hline Acute kidney injury & $163(3.2 \%)$ & $163(3.2 \%)$ & 1.01 & $0.72-1.42$ & 0.93 \\
\hline Dural tear & $0(0.0 \%)$ & $0(0.0 \%)$ & NA & NA & NA \\
\hline CSF leak & $7(0.1 \%)$ & $8(0.2 \%)$ & 0.82 & $0.19-3.21$ & 0.75 \\
\hline Cerebral infarction & $34(0.7 \%)$ & $31(0.6 \%)$ & 1.01 & $0.50-2.07$ & 0.97 \\
\hline \multirow[t]{2}{*}{ Mortality } & $137(2.7 \%)$ & $159(3.2 \%)$ & 1.13 & $0.80-1.61$ & 0.48 \\
\hline & Frail Patients, $n=3,378$ & Propensity-Matched Nonfrail Patients, $n=3,325$ & OR & $95 \% \mathrm{Cl}$ & $p$ Value \\
\hline \multicolumn{6}{|l|}{ 180-day readmission } \\
\hline Readmission rate & $1,416(41.9 \%)$ & $1,457(43.8 \%)$ & 0.95 & $0.83-1.10$ & 0.50 \\
\hline Infection & $74(2.2 \%)$ & $65(2.0 \%)$ & 1.27 & $0.78-2.06$ & 0.33 \\
\hline Thromboembolism & $176(5.2 \%)$ & $188(5.7 \%)$ & 0.94 & $0.69-1.29$ & 0.72 \\
\hline Acute kidney injury & $162(4.8 \%)$ & $134(4.0 \%)$ & 1.15 & $0.81-1.64$ & 0.43 \\
\hline Dural tear & $0(0.0 \%)$ & $0(0.0 \%)$ & NA & NA & NA \\
\hline CSF leak & $5(0.1 \%)$ & $6(0.2 \%)$ & 0.78 & $0.14-3.74$ & 0.73 \\
\hline Cerebral infarction & $34(1.0 \%)$ & $15(0.5 \%)$ & 2.06 & $0.89-5.16$ & 0.087 \\
\hline Mortality & $121(3.6 \%)$ & $115(3.5 \%)$ & 1.21 & $0.83-1.75$ & 0.32 \\
\hline
\end{tabular}

Boldface type indicates statistical significance.

to nonfrail patients (7.1 days), may be more accurate in demonstrating differences between the two cohorts than the 30-day readmission rates.

In the current neurosurgical setting, comparable management techniques are implemented for both frail and nonfrail patients. However, acute postoperative outcomes for patients meeting JHACG criteria for frailty may be improved by implementing preoperative support strategies. Prior studies have demonstrated that functional physical decline in frail patients may be counteracted through physical therapy, exercise training, and nutrient supplementation. ${ }^{35-37}$ In addition to addressing physical decline, preoperative social support through consultations with social workers, assessments of cognitive function, and proper patient counseling may offer an opportunity to address the social, emotional, and behavioral aspects of patient frailty. ${ }^{35,38,39}$ By implementing holistic medical review and patient-tailored management prior to neurosurgery, it may be possible to reduce the high acute postsurgical complication rates seen in frail patients.

\section{Limitations of the Study}

This study has several limitations. First, there are inherent limitations associated with a retrospective database study, including a dependence on the quality and depth of the records kept for patients included in this study. As with all databases, there is a risk of selection bias, which we aimed to minimize by using random methods when selecting subgroups, including large sample sizes, and propensity score matching for demographics and comorbidities. Second, this study includes years prior to and after the adoption of ICD-10 coding. As a result, both ICD-9 and ICD-10 codes had to be used to query patients and complications. Third, the NRD encapsulates 28 states and roughly $60 \%$ of the United States population, so the conclusions drawn in this study may not be applicable to patients from certain geographic regions. However, few databases-all of which contain far fewer patient files than the NRD-are able to achieve even this degree of geographic coverage, with many providing data from only a handful of states. In addition, the use of discharge weights provided with the NRD 
allow for nationally representative trends and conclusions. Although a large database can identify causality, predict hazards, and improve care quality, it is limited in terms of volume and variety. Specifically, metrics of accuracy, adequacy, reporting sources, and missing data may influence the quality of analysis possible through a database. Rigorous inclusion and exclusion criteria are therefore necessary to ensure high-quality analysis. Last, ICD-9 and ICD-10 coding prevented additional specificity with regard to exact neoplasm subtype. We addressed this limitation by using the most granular codes available to us and reporting the rates of benign and malignant primary CNS neoplasms.

\section{Conclusions}

The short- and long-term effects of frailty on geriatric patients with primary CNS neoplasms have not been well studied, preventing evidence-based advancements in neurosurgical management. Our findings suggest that frail patients are at a higher risk of acute short-term postoperative complications, including increased LOS, cost, anemia, infection, kidney injury, cerebral infarction, and UTI. However, long-term analysis at 90- and 180-day readmission intervals revealed no statistically significant difference in complication rates between frail and nonfrail cohorts with the same diagnoses and procedures. Further research is necessary to fully understand the role of frailty on perioperative neurosurgical outcomes in geriatric patients.

\section{References}

1. Xue Q-L. The frailty syndrome: definition and natural history. Clin Geriatr Med. 2011;27(1):1-15.

2. Bandeen-Roche K, Xue Q-L, Ferrucci L, et al. Phenotype of frailty: characterization in the women's health and aging studies. J Gerontol A Biol Sci Med Sci.2006;61(3):262-266.

3. Fried LP, Tangen CM, Walston J, et al. Frailty in older adults: evidence for a phenotype. J Gerontol A Biol Sci Med Sci. 2001;56(3):M146-M156.

4. Gill TM, Gahbauer EA, Allore HG, Han L. Transitions between frailty states among community-living older persons. Arch Intern Med. 2006;166(4):418-423.

5. Graham JE, Snih SA, Berges IM, et al. Frailty and 10-year mortality in community-living Mexican American older adults. Gerontology. 2009;55(6):644-651.

6. Ensrud KE, Ewing SK, Cawthon PM, et al. A comparison of frailty indexes for the prediction of falls, disability, fractures, and mortality in older men. J Am Geriatr Soc. 2009;57(3): 492-498.

7. Clegg A, Young J, Iliffe S, et al. Frailty in elderly people. Lancet. 2013;381(9868):752-762.

8. Ali R, Schwalb JM, Nerenz DR, et al. Use of the modified frailty index to predict 30-day morbidity and mortality from spine surgery. J Neurosurg Spine. 2016;25(4):537-541.

9. Cloney M, D'Amico R, Lebovic J, et al. Frailty in geriatric glioblastoma patients: a predictor of operative morbidity and outcome. World Neurosurg. 2016;89:362-367.

10. Ethun CG, Bilen MA, Jani AB, et al. Frailty and cancer: Implications for oncology surgery, medical oncology, and radiation oncology. CA Cancer J Clin. 2017;67(5):362-377.

11. Joseph B, Pandit V, Sadoun M, et al. Frailty in surgery. $J$ Trauma Acute Care Surg. 2014;76(4):1151-1156.

12. Leven DM, Lee NJ, Kothari P, et al. frailty index is a significant predictor of complications and mortality after surgery for adult spinal deformity. Spine (Phila Pa 1976). 2016; 41(23):E1394-E1401.
13. Tomlinson SB, Piper K, Kimmell KT, Vates GE. Preoperative frailty score for 30-day morbidity and mortality after cranial neurosurgery. World Neurosurg. 2017;107:959-965.

14. Asemota AO, Gallia GL. Impact of frailty on short-term outcomes in patients undergoing transsphenoidal pituitary surgery. J Neurosurg. 2019;132(2):360-370.

15. The Johns Hopkins Adjusted Clinical Groups Technical Reference Guide, Version 9.0. Johns Hopkins University; 2009.

16. Sternberg SA, Bentur N, Abrams C, et al. Identifying frail older people using predictive modeling. Am J Manag Care. 2012;18(10):e392-e397.

17. Abrams C, Lieberman R, Weiner JP. Development and Evaluation of The Johns Hopkins University Risk Adjustment Models for Medicare+Choice Plan Payment. Johns Hopkins University; 2003.

18. Charlson Comorbidity Index (CCI) Calculator. Accessed August 17, 2020. https://www.thecalculator.co/health/CharlsonComorbidity-Index-(CCI)-Calculator-765.html

19. Charlson M, Szatrowski TP, Peterson J, Gold J. Validation of a combined comorbidity index. J Clin Epidemiol. 1994; 47(11):1245-1251.

20. Charlson ME, Pompei P, Ales KL, MacKenzie CR. A new method of classifying prognostic comorbidity in longitudinal studies: development and validation. J Chronic Dis. 1987; 40(5):373-383.

21. Quan $\mathrm{H}, \mathrm{Li} \mathrm{B}$, Couris CM, et al. Updating and validating the Charlson comorbidity index and score for risk adjustment in hospital discharge abstracts using data from 6 countries. Am J Epidemiol. 2011;173(6):676-682.

22. Radovanovic D, Seifert B, Urban P, et al. Validity of Charlson Comorbidity Index in patients hospitalised with acute coronary syndrome. Insights from the nationwide AMIS Plus registry 2002-2012. Heart. 2014;100(4):288-294.

23. Ho D, Imai K, King G, Stuart E. MatchIt: nonparametric preprocessing for parametric causal inference. J Stat Softw. 2011;42(8):1-28.

24. Youngerman BE, Neugut AI, Yang J, et al. The modified frailty index and 30-day adverse events in oncologic neurosurgery. J Neurooncol. 2018;136(1):197-206.

25. Harland TA, Wang M, Gunaydin D, et al. Frailty as a predictor of neurosurgical outcomes in brain tumor patients. World Neurosurg. 2020;133:e813-e818.

26. Miller EK, Neuman BJ, Jain A, et al. An assessment of frailty as a tool for risk stratification in adult spinal deformity surgery. Neurosurg Focus. 2017;43(6):E3.

27. Salcman M. Brain tumors and the geriatric patient. J Am Geriatr Soc. 1982;30(8):501-508.

28. Lowry JK, Snyder JJ, Lowry PW. Brain tumors in the elderly: recent trends in a Minnesota cohort study. Arch Neurol. 1998; 55(7):922-928.

29. Krishnan M, Beck S, Havelock W, et al. Predicting outcome after hip fracture: using a frailty index to integrate comprehensive geriatric assessment results. Age Ageing. 2014;43(1): 122-126.

30. Kenig J, Zychiewicz B, Olszewska U, et al. Six screening instruments for frailty in older patients qualified for emergency abdominal surgery. Arch Gerontol Geriatr. 2015;61(3): 437-442.

31. Kristjansson SR, Rønning B, Hurria A, et al. A comparison of two pre-operative frailty measures in older surgical cancer patients. J Geriatr Oncol. 2012;3(1):1-7.

32. Sündermann S, Dademasch A, Praetorius J, et al. Comprehensive assessment of frailty for elderly high-risk patients undergoing cardiac surgery. Eur J Cardiothorac Surg. 2011; 39(1):33-37.

33. Stortecky S, Schoenenberger AW, Moser A, et al. Evaluation of multidimensional geriatric assessment as a predictor of mortality and cardiovascular events after transcatheter aor- 
tic valve implantation. JACC Cardiovasc Interv. 2012;5(5): 489-496.

34. Hewitt J, Moug SJ, Middleton M, et al. Prevalence of frailty and its association with mortality in general surgery. Am J Surg. 2015;209(2):254-259.

35. Chan SP, Ip KY, Irwin MG. Peri-operative optimisation of elderly and frail patients: a narrative review. Anaesthesia. 2019;74(suppl 1):80-89.

36. Fiatarone MA, O’Neill EF, Ryan ND, et al. Exercise training and nutritional supplementation for physical frailty in very elderly people. N Engl J Med. 1994;330(25):1769-1775.

37. Gill TM, Baker DI, Gottschalk M, et al. A program to prevent functional decline in physically frail, elderly persons who live at home. N Engl J Med. 2002;347(14):1068-1074.

38. Turner G, Clegg A. Best practice guidelines for the management of frailty: a British Geriatrics Society, Age UK and Royal College of General Practitioners report. Age Ageing. 2014;43(6):744-747.

39. Bunt S, Steverink N, Olthof J, et al. Social frailty in older adults: a scoping review. Eur J Ageing. 2017;14(3):323-334.

\section{Disclosures}

The authors report no conflict of interest concerning the materials or methods used in this study or the findings specified in this paper.

\section{Author Contributions}

Conception and design: Shahrestani, Lehrich, RC Ransom. Acquisition of data: Shahrestani, Chen. Analysis and interpretation of data: Shahrestani, Ballatori. Drafting the article: Sahyouni, Shahrestani, Ballatori, Ton. Critically revising the article: Sahyouni, Lehrich, Tafreshi, Brown, Lien, S Ransom, RC Ransom, Ballatori, Ton, Chen. Reviewed submitted version of manuscript: Sahyouni, Lehrich, Tafreshi, Brown, Lien, S Ransom, RC Ransom, Chen. Statistical analysis: Shahrestani. Administrative/technical/material support: Tafreshi, S Ransom, Ballatori, Ton. Study supervision: Sahyouni, Shahrestani, Lehrich, Tafreshi, Brown, Lien, RC Ransom, Ballatori.

\section{Supplemental Information}

Online-Only Content

Supplemental material is available online.

Supplementary Table 1. https://thejns.org/doi/suppl/10.3171/ 2020.7.FOCUS20426.

\section{Correspondence}

Ronald Sahyouni: University of California, San Diego, CA. rsahyoun@uci.edu. 